\title{
Benign Osteoblastoma of the Sphenoid Bone
}

\author{
Mavilde Arantes, M.D., ${ }^{1}$ Mário Resende, M.D., ${ }^{2}$ Mrinalini Honavar, M.D., ${ }^{3}$ \\ Manuel Melo Pires, M.D., 4 Jorge Resende Pereira, M.D., 1 \\ and Artur Rocha Vaz, M.D. ${ }^{2}$
}

Osteoblastoma is an uncommon benign bone tumor that accounts for $1 \%$ of all primary bone tumors. Well documented in the spine and long bones, it is rarely found in the skull, namely in the sphenoid bone, with only five cases reported in the literature. We report a case of an 11-year-old girl with a histologically confirmed benign osteoblastoma in an unusual location and an atypical aspect on the imaging studies.

KEYWORDS: Benign osteoblastoma, child, sphenoid bone, radical resection

Benign osteoblastoma is an uncommon, solitary, bone-producing tumor characterized by richly vascular, loose fibrous stroma in which osteoid is deposited, rimmed by prominent osteoblasts and osteoclasts. ${ }^{1}$ It most often involves the vertebral column and long bones. ${ }^{2}$ The tumor develops rarely in the skull, where it has a preference for the temporal and frontal bones. ${ }^{3} \mathrm{We}$ present a case of an 11-year-old girl with a benign osteoblastoma in an unusual localization and an atypical aspect on the imaging studies. Radical resection produced an excellent result.

\section{CASE REPORT}

An 11-year-old girl was admitted with a 3-month history of moderate frontal headaches associated with gradually increasing painless proptosis of the right eye. She had no history of trauma or surgery, and her medical records were unremarkable. On admission, the patient had no neurological deficit, except for the right proptosis. Routine blood examination was normal. Axial bone-target computed tomography (CT) showed a destructive, heterogeneous bony mass involving

(e-mail: mavildearantes@hotmail.com)

Skull Base 2009;19:437-442. Copyright (C) 2009 by Thieme Medical Publishers, Inc., 333 Seventh Avenue, New York, NY 10001, USA. Tel: +1 (212) 584-4662.

Received: September 7, 2008. Accepted: December 29, 2008. Published online: June 5, 2009.

DOI 10.1055/s-0029-1224861. ISSN 1531-5010. 


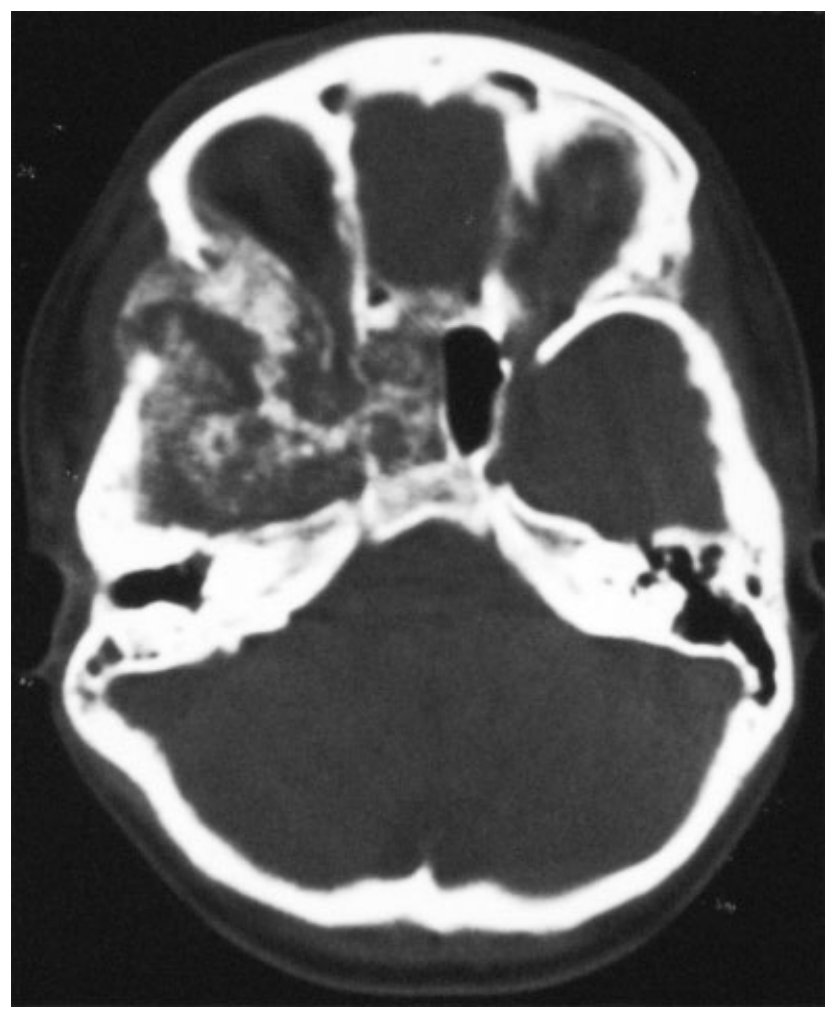

Figure 1 Axial bone-target computed tomography scan showed a bony destructive heterogenous mass that invaded the sphenoid bone with right anterior and middle cranial fossa extension.

the sphenoid bone extending posteroinferiorly into the middle cranial fossa and anteriorly into the orbital and maxillary region (Fig. 1). Magnetic resonance imaging (MRI) revealed a heterogeneous mass, isointense on T1- and T2weighted images with some small hypointense areas (Fig. 2A and B) and great enhancement after the administration of gadolinium (Fig. 2C). MRI was also important in the evaluation of the tumor extension, demonstrating invasion of the sphenoidal sinus and body, the tuberculum sellae, the right anterior clinoid process, the right greater and lesser sphenoid wing, the right superior orbital fissure, the right optic canal, the posterior portion of the roof of the right orbit, the right posterior ethmoid complex, the right maxillary sinus and right pterygoid fossa, with posterior displacement of the temporal pole. The lesion measured $6.2 \times 4.3 \times 3.9 \mathrm{~cm}$. The patient was submitted to surgery by the senior author. A right-sided frontotemporal approach with extradural exposure of the anterior skull base was made. Opening of the muscular layer revealed tumor directly beneath it. The bone was replaced by firm fibrous tissue with a gritty texture. The tumor appeared to be richly vascular and bled heavily. Complete macroscopic removal of the lesion was achieved. Histopathological findings showed the lesion was composed of irregular deposits of osteoid and a network of coarsely formed osteoid trabeculae surrounded by plump osteoblasts and occasional osteoclasts. In places, the osteoblasts rimmed the bony trabeculae, and in other places, well-formed woven bone was noted. Elsewhere loose richly vascular fibroblastic stroma was seen with fine deposits of osteoid (Fig. 3). There was no evidence of malignancy. The features were those of a benign osteoblastoma. In the immediate postoperative period, the patient had decreased visual acuity in 

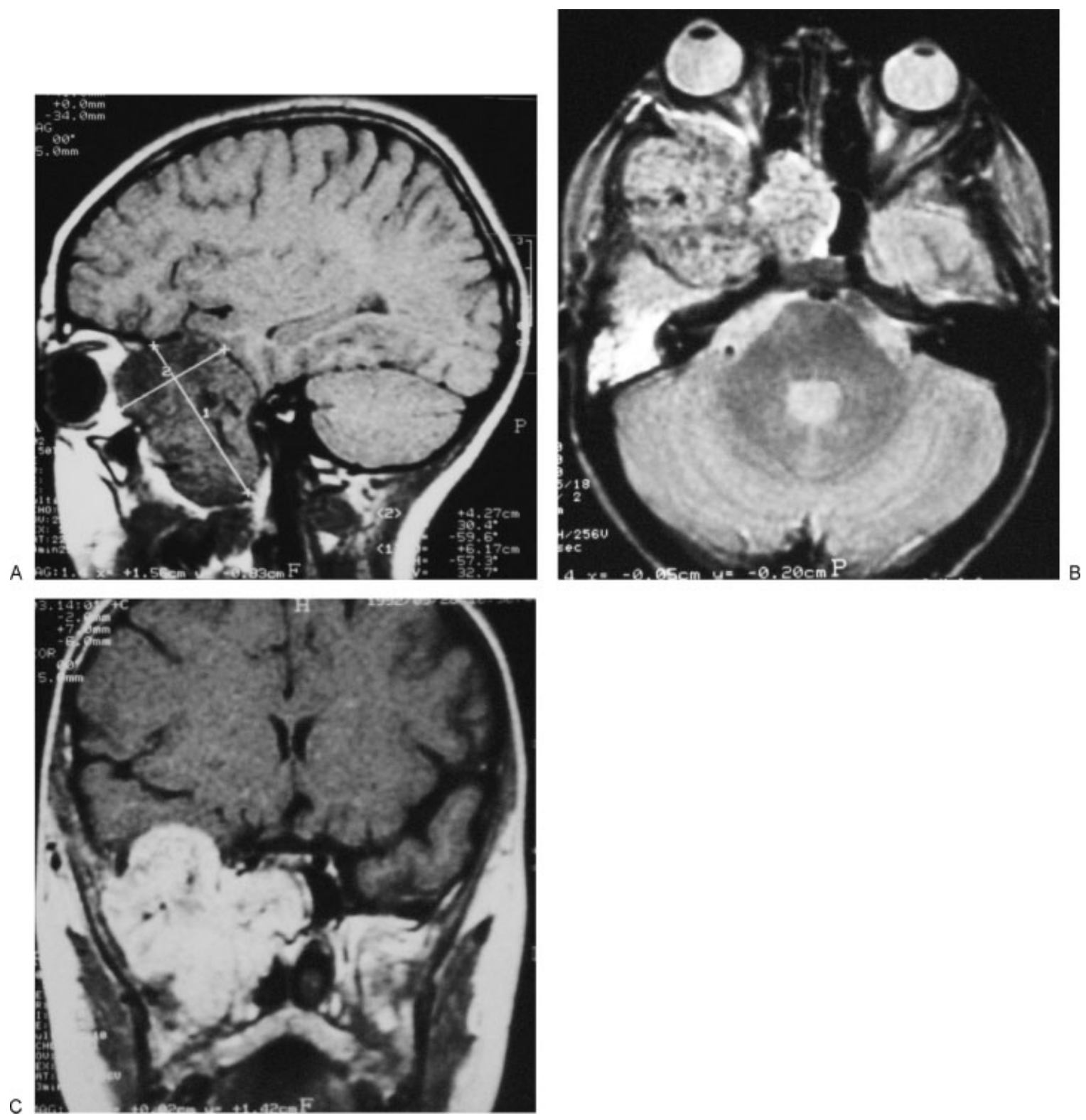

Figure 2 Magnetic resonance imaging showing (A) a sagittal T1-weighted image, (B) an axial T2-weighted image with a heterogeneous mass, predominantly isointense but with some small hypointense areas, and (C) a coronal image with great enhancement after the administration of gadolinium.

the right eye, with progressive recovery and no deficit a week later. Her proptosis resolved rapidly. Postoperative CT images showed no residual lesion. Fifteen years postoperatively, the patient is well, with no neurological deficit and no imagiologic recurrence of the tumor (Fig. 4).

\section{DISCUSSION}

The term "benign osteoblastoma" was independently proposed by Jaffe (1956) and Lichtenstein (1956) to define a vascular osteoid and bone-forming tumor containing numerous osteoblasts and rich vascularized delicate fibrous stroma with a benign 


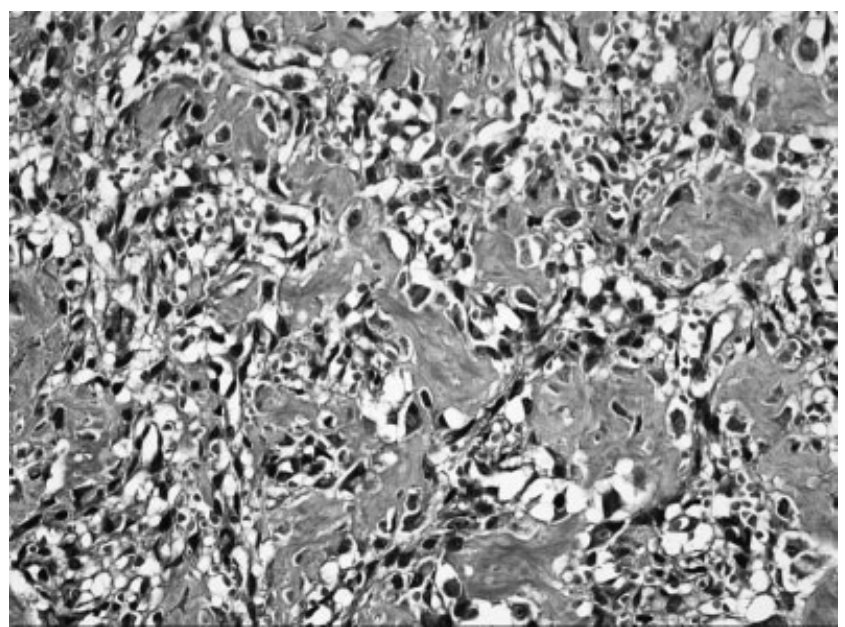

Figure 3 Photomicrograph of the resected lesion showing haphazardly deposited osteoid surrounded by plump osteoblasts.

appearance. ${ }^{4}$ Benign osteoblastoma is a rare tumor that constitutes $\sim 1 \%$ of all primary bone tumors and $3 \%$ of all benign bone tumors. ${ }^{5}$ Osteoblastomas usually occur in patients $<30$ years of age with a peak incidence in the second decade of life and a range of 3 to 78 years. The tumor shows a predilection for the male gender. It is most commonly located in the vertebral column and metaphysis or

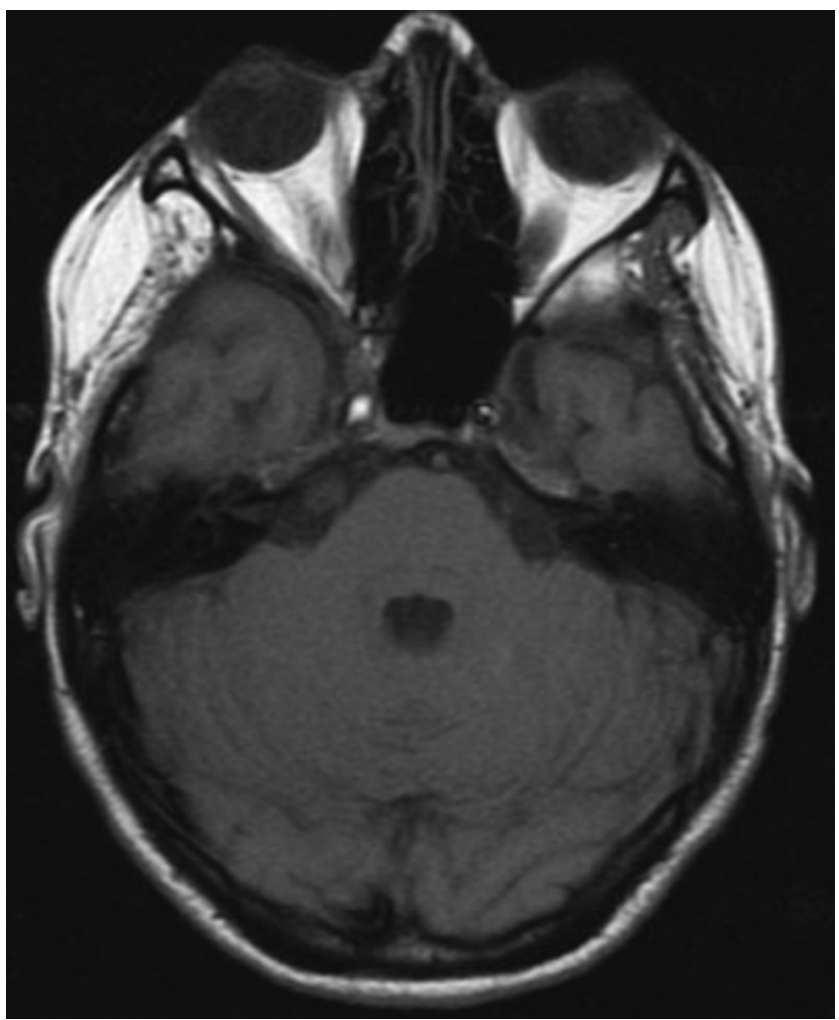

Figure 4 Postoperative image showed no residual lesion. 
diaphysis of long bones. It rarely develops in the skull, but when it does, it has a preference for the temporal and frontal bones. ${ }^{6,7}$ Its occurrence in the sphenoid bone is exceptional. ${ }^{8-11}$ It is a painful tumor and usually grows slowly, becoming symptomatic only when it invades important structures, such as the cranial nerve foramina. Neuroradiological examination, namely with $\mathrm{CT}$ and MRI, is particularly important to make a preoperative diagnosis. Besides, it is essential to define the nature and extent of soft tissue involvement or compromise. The typical image is a "lytic" zone surrounded by bony condensation and a minimal osteosclerotic reaction peripherally, rarely invading the soft tissues. Generally, CT reveals a bony destruction and focal calcification with variable patterns of contrast enhancement. MRI is characterized by a hypointense or isointense mass on a T1weighted image with homogeneous or heterogeneous enhancement after the administration of gadolinium and by a hypointense mass on a T2-weighted image, unlike our case that showed a heterogeneous lesion, with some small areas that have low signal intensity on T1- and T2-weighted images, suggesting a mixture of bone and fibrous tissue. ${ }^{12}$ Benign osteoblastoma should be differentiated from other similar bone-forming tumors, such as osteomas, osteoid osteomas, osteosarcoma, ossifying fibroma, calcifying meningioma, giant cell tumor, aneurysmal bone cyst, and fibrous dysplasia. ${ }^{13}$

Definitive diagnosis is made by histological examination that reveals osteoblast-like cells disseminated in an abundant conjunctive background surrounded by immature richly vascularized bone. No malignant features such as anaplasia, necrosis, or abnormal mitosis are found. The majority of the authors currently recommended that benign osteoblastoma, despite its benign biological behavior, should be treated with total resection of the tumor. Prognosis after complete removal is uniformly good. ${ }^{14}$ There is no definitive role for adjuvant radiotherapy or chemotherapy, and radiation therapy currently is reserved for specific cases in which only subtotal resection has been achieved or for unresectable lesions in symptomatic patients. To our concern, this is the fifth reported case of benign osteoblastoma in the sphenoid bone. MRI proved to be effective for the evaluation of the intracranial and intraosseous extensions of the tumor. The mass was totally excised and no recurrence was observed, for at least 15 years, confirming that total surgical resection is the treatment of choice of benign osteoblastoma.

\section{REFERENCES}

1. Cervoni L, Innocenzi G, Raguso M, Salvati M, Caruso R. Osteoblastoma of the calvaria: report of two cases diagnosed with MRI and clinical review. Neurosurg Rev 1997;20(1):51-54

2. Moon KS, Jung S, Lee JH, Jung T, Kim IY, Kang SS. Benign osteoblastoma of the occipital bone: case report and literature review. Neuropathology 2006;26(2):141-146

3. Miyazaki S, Tsubokawa T, Katayama Y, Kai Y, Sakurai I. Benign osteoblastoma of the temporal bone of an infant. Surg Neurol 1987;27(3):277-283

4. Jaffe HL. Benign osteoblastoma. Bull Hosp Joint Dis 1956;17(2):141-151

5. Lichtenstein L. Benign osteoblastoma: a category of ostead and bone-forming tumours other than classical osteoid which may be mistaken for giant-cell tumour or osteogenic sarcoma. Cancer 1956;9:1044-1052

6. Choudhury AR, al Amin MS, Chaudhri KA, al Moutaery KR. Benign osteoblastoma of the parietal bone. Childs Nerv Syst 1995;11(2):115-117

7. Doron Y, Gruszkiewicz J, Gelli B, Peyer E. Benign osteoblastoma of vertebral column and skull. Surg Neurol 1977;7(2):86-90

8. Ciappetta P, Salvati M, Raco A, Artico M, Gagliardi FM. Benign osteoblastoma of the sphenoid bone. Neurochirurgia (Stuttg) 1991;34(3):97-100

9. Berciano J, Pérez-López JL, Fernández F, Val F, Leno C. Voluminous benign osteoblastoma of the skull. Surg Neurol 1983;20(5):383-386

10. Contreras F, Segura A. Osteoblastoma benign del esfenoides [in Spanish]. Revista Espanola de Patologia 1968;1:65-70

11. Derome P, Akerman M, Anquez L. Les tumeurs sphenoethmoidales. Possibilités d'exérèse et réparation chirurgicales. Rapport de la Société de Neurochirurgia de Langue Française [in French]. Neurochirurgie 1972;15(Suppl 1): 136-139

12. Bilkay U, Erdem O, Ozek C, et al. A rare location of benign osteoblastoma: review of the literature and report of a case. J Craniofac Surg 2004;15(2):222-225

13. Heuck A, Stäbler A, Wörtler K, Steinborn M. Benign bone-forming tumors. Radiologe 2001;41(7):540-547

14. Doshi SV, Frantz TD, Korol HW. Benign osteoblastoma of the temporal bone: case report and literature review. Am J Otolaryngol 2001;22(3):211-214 